\title{
Research on the Integrated Control Technology of End-to-end Business Detection Management Heterogeneous System
}

\author{
WANG Chun-ying, LI Wen-cui, AN Zhi-yuan, SHU Xin-jian, WU Li-jie, ZHANG \\ Ning-ning
}

Keywords: the terminal communication access network, end-to-end business detection management heterogeneous, the integrated control technology

\begin{abstract}
The integrated control technology on end-to-end business detection management heterogeneous of the terminal communication access network, is the technology which actively promote its current function while optimizing the Access Network module of communication resource management system. This paper puts forward the whole business communication management of intelligent distribution and electricity communication demand and the application of heterogeneous cross network, which provide good application support and user experience for the operation, management and decision making of all departments.
\end{abstract}

\section{Introduction}

The telecom business support system built by foreign public network operators usually includes the network automatic activation function, which can realize the automatic configuration of the business according to the resource allocation scheme. NGOSS, the next generation of operating software systems, is a system of operational support systems proposed by the TMF (Tele-Management Forum), which ensure operation support system standardization, gradually deepened, interconnection operation, implementation end-to-end management and highly automated from four aspects: rules (plug and play), process (business process model), information (public data correlation processing) and production [1].

At present, with the development trend of network activation system of foreign public network operators, the network activation system will provide the encapsulation of the automatic network activation interface of the entire network, system and business platform in terms of business competencies. Provide automatic network activation service ability which is full-web, full-service, unified, and centralized, and ensure the real-time and effectiveness of the automated network activation service capabilities, and bring real-time, automated and centralized customer experience value to telecom operators.

With the advent of the era of all business operations, it is urgent to introduce the integrated business with competitive advantages to meet the changing needs of the market. Network activation is the process of completing the end-to-end configuration of the service information ordered by the customer in the network device, which is the mapping of customer service to the actual physical network.

Public network operators aims for improving the speed of service, reducing the cycle of the new business launch [2], shielding the differences of each manufacturer network equipment in the service configuration process, established a comprehensive network activation system to satisfy the requirement of the automation and real-time business to activate. After years of construction, the integrated network activation system has evolved into the third generation.

Although the research and application of integrated network activation system of the public network operators mainly concentrated in SPC business, mobile business, intelligent network business, NGN business, Internet business, value-added services, etc, which has certain difference with the electric power communication business. But its technical principle and software implementation has a certain universality, which provides the necessary reference for the objective of the study and reference [3-4]. 


\section{The Method of Big Data Strict Management}

The method of big data strict management, which can solve problems currently faced, such as, data standard, data quality issues, meta-data management and data services, is based on the terminal communication access network platform to improve the data information management ability from the aspects of system, standard, monitoring and process. The data governance model architecture is shown as Fig.1.

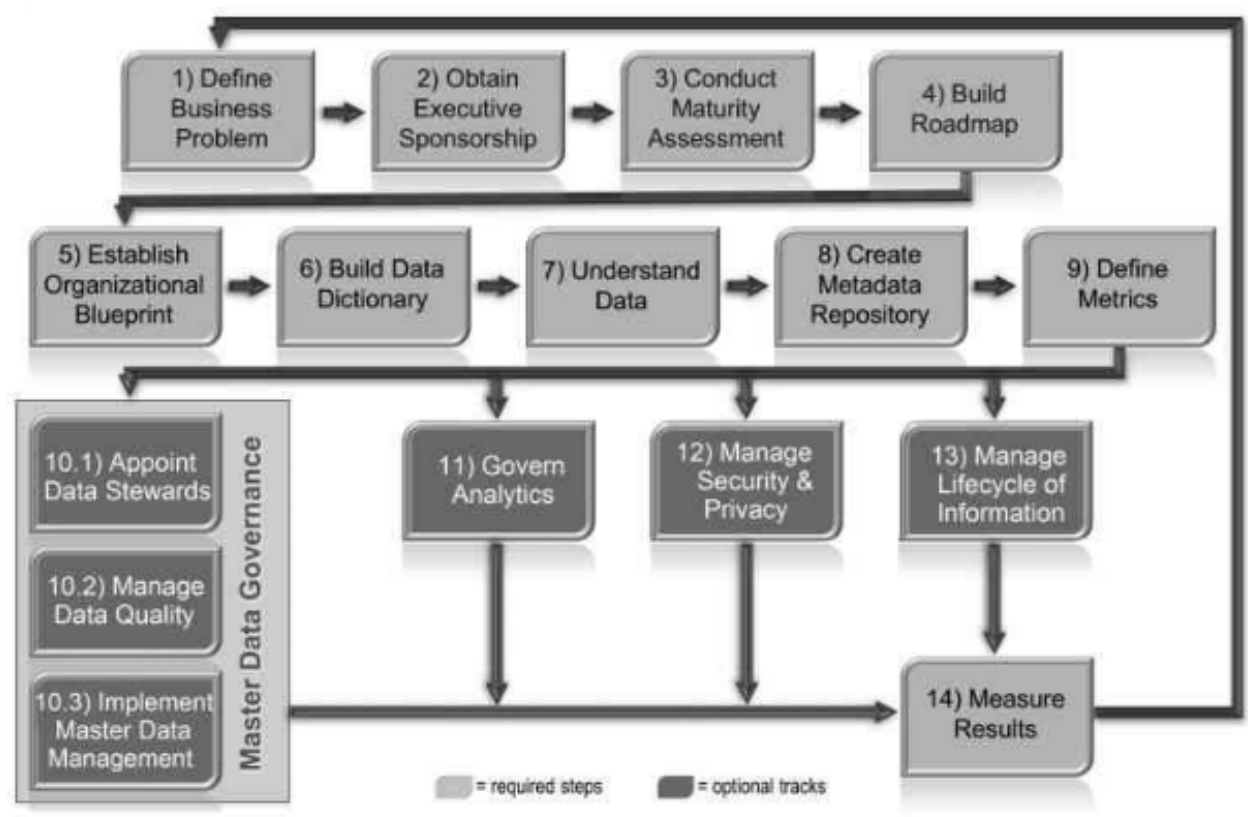

Fig.1 Data governance model architecture

(1) Standardization of data standards: Standardized management forms the basis of big data platforms, including data control system and process specification document, information definition, etc.

(2) Data relationship contextualized: the influence and blood analysis of data transfer, dependence and relationship.

(3) Data quality quantified: Manage the data quality of the big data platform comprehensively, to realize data quality check, dimensional analysis and problem tracking.

(4) Data service digitization: Provides service communication channels for business users for data platforms.

\section{Research on the Whole Business Communication Model of Access Network}

Do fully investigate on the operation of the distribution network, and the informational requirements of the future distribution network. And then the business is divided into different groups according to the different requirements of communication. And there is a comprehensive in-depth analysis study on the modeling of the network principles, methods, and verification methods, to sum up the shortage of the electric network and the future focus, which are used to build all business communication models [5].

By analyzing the business data and data flow of the node, the communication demand of complex intelligent power distribution is studied, and the whole business communication model of intelligent configuration is constructed. The business logic diagram of terminal communication access network is shown below, as Fig. 2 . 


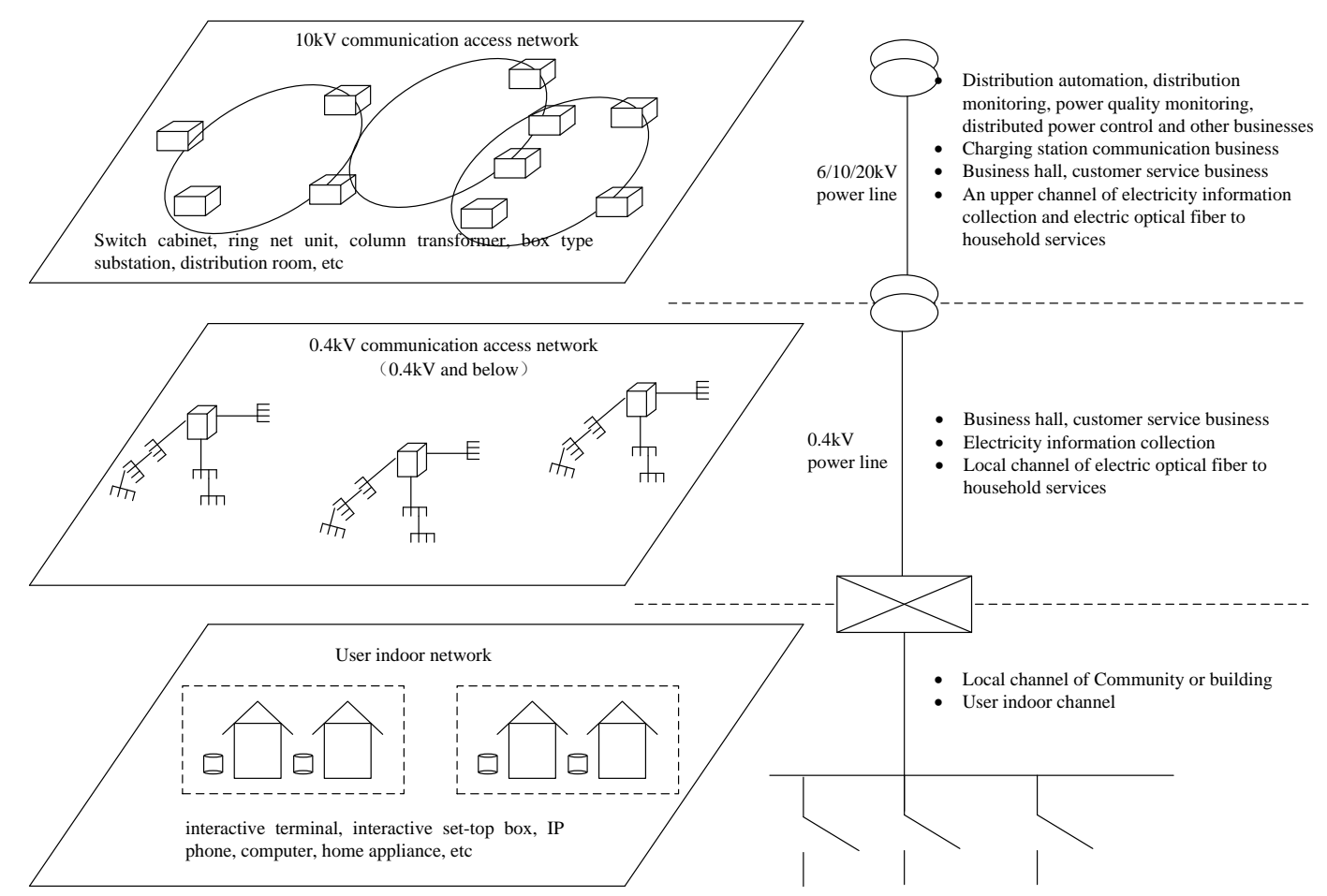

Fig.2 The business logic diagram of terminal communication access network

\section{Research on the End-to-end Full Network Model of Backbone Network and Access Network}

At present, the failure of the access network may be: terminal equipment, access network equipment or media, switch, transmission equipment or channel. Therefore, in the case of specific business failure, all aspects of the end-to-end process should be involved. Troubleshooting can be achieved through collaboration between communication professional and other business departments. By the use of communication management system, combining with the established management system of other relevant departments, such as the production and distribution system, the data base and technical conditions of the end-to-end model research have been developed.

The Whole Road Mainly Composed of PON (Including Mixed Network). Terminal equipment $\longleftrightarrow \longrightarrow$ Wireless or carrier slave equipment $\longleftrightarrow \longrightarrow$ Wireless or carrier master equipment $\longleftrightarrow \longrightarrow$ ONU equipment $\longrightarrow$ ODN/optical splitter $\longrightarrow$ OLT $\longleftrightarrow$ Switch $\longrightarrow$ the GE interface of backbone transmission equipment $\longleftrightarrow$ Backbone transmission channel $\longleftrightarrow$ the GE interface of Main station transmission equipment $\longleftrightarrow$ Switch $\longrightarrow$ Main station business system. The hybrid network of optical fiber and wireless network is shown as Fig.3.

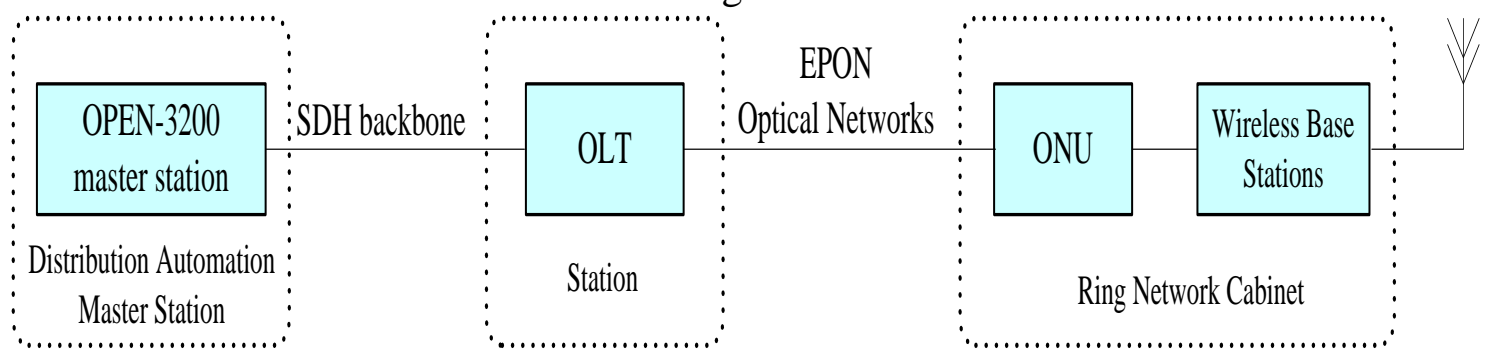

Fig.3 The hybrid network of optical fiber and wireless network

The Whole Road Mainly Composed of PLC. Terminal equipment $\longleftrightarrow$ Carrier slave equipment $\longleftrightarrow$ power line $\longrightarrow$ carrier master equipment $\longleftrightarrow$ Communication manager $\longleftrightarrow \longrightarrow$ Switch $\longleftrightarrow$ the GE interface of backbone transmission equipment $\longrightarrow$ Backbone transmission channel $\longleftrightarrow$ the GE interface of main station transmission equipment $\longleftrightarrow$ Switch $\longleftrightarrow$ Main station business system.

The Whole Road Mainly Composed of Wireless. Terminal equipment $\longleftrightarrow$ Wireless slave equipment $\longleftrightarrow$ wireless network or public network $\longrightarrow$ Wireless master equipment $\longleftrightarrow$ Communication manager $\longleftrightarrow$ Switch $\longrightarrow$ the GE interface of backbone transmission equipment 
$\longleftrightarrow$ Backbone transmission channel $\longrightarrow$ the GE interface of Main station transmission equipment $\longleftrightarrow$ Switch $\longrightarrow$ Main station business system. The hybrid network of optical fiber and carrier is shown as Fig.4.

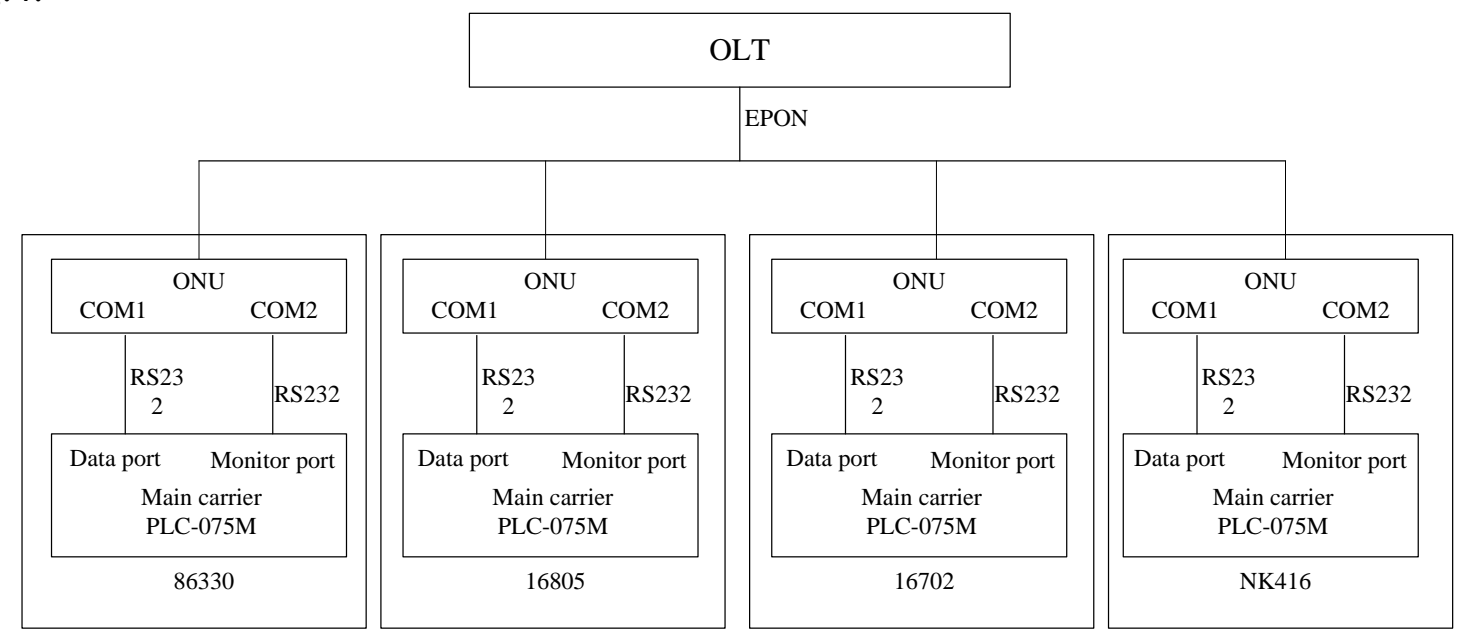

Fig. 4 The hybrid network of optical fiber and carrier

\section{Conclusion}

The integrated control technology on End-to-end business detection management heterogeneous of the terminal communication access network is the technology which actively promotes its current function while optimizing the Access Network module of communication resource management system. In this paper, according to the requirements of relevant standards, the communication access network business management system model was constructed in general, clear and correct data format. Establish the whole business communication model, the end-to-end entire network model and the whole railway network of the communication terminal access so as to achieve end-to-end business detection management comprehensive control function of the heterogeneous applications.

\section{References}

[1] WEI Yu-fan. Design and Realization of Equipment Maintenance Information Management and Analysis System [J].Computer and Information Technology, 2009,17(4):62-66

[2] Li Wencui, Li Xiong, Zhang Chi, Wu Lijie, Shu Xinjian, Tang Weixia. Research on the Figure Module Integration Technology in Communication Monitoring. ICEEECS, 2016(50), 686-689.

[3] Li Wencui, Li Xiong, Yang Ying, Ding Ying, Shu Xinjian, Zhang Yong.

[4] A Brief Talk on Information and Communication Safety Management of Electric Power Enterprise. ICMMBE, 2016(83), 217-220.

[5] Li Wencui, Shu xinjian, Li Xiong, Gao Hui, Liu Bo, Wang Chunying, Yang Ying. An Information Security ManagementSystem Based on "Five-in-one”. ICEEECS, 2016(50), 743-745. 